\title{
Retraction: Graded bandgap perovskite solar cells
}

\author{
Onur Ergen, S. Matt Gilbert, Thang Pham, Sally J. Turner, Mark Tian Zhi Tan, Marcus A. Worsley and Alex Zettl
}

Nature Materials 16, 522-525 (2017); published online 7 November 2016; retracted after print 20 December 2017.

The authors of the study are retracting this Letter due to concerns about the reproducibility of the photovoltaic architecture performance presented, and with the interpretation of the data included in the manuscript and Supplementary Information. This Letter presented solar cells based on two perovskite layers separated by a monolayer of hexagonal boron nitride, deposited on graphene aerogel. The large variability of the current-voltage and spectral response of nominally identical devices and the rapid time evolution of key photovoltaic parameters undermine the authors' confidence in the conclusions that can be drawn at this stage regarding the performance of these architectures, and they therefore wish to retract the Letter. All the authors agree with the retraction.

\section{Corrigendum: Beating the thermodynamic limit with photo-activation of $n$-doping in organic semiconductors}

Xin Lin, Berthold Wegner, Kyung Min Lee, Michael A. Fusella, Fengyu Zhang, Karttikay Moudgil, Barry P. Rand, Stephen Barlow, Seth R. Marder, Norbert Koch and Antoine Kahn

Nature Materials 16, 1209-1215 (2017); published online 13 November 2017; corrected after print 15 December 2017.

In the version of this Article originally published, the source of 'ZADN' stated in the Methods should have read 'obtained as free research samples from Guangzhou ChinaRay Optoelectronic Materials' instead of 'China-Ray'. This has now been corrected in the online versions of the Article.

\section{Corrigendum: Plasmonic nanosensors with inverse sensitivity by means of enzyme-guided crystal growth}

\author{
Laura Rodríguez-Lorenzo, Roberto de la Rica, Ramón A. Álvarez-Puebla, Luis M. Liz-Marzán and Molly M. Stevens
}

Nature Materials 11, 604-607 (2012); published online 27 May 2012; corrected after print 15 December 2017.

In the version of this Letter originally published, the $x$ and $y$ values of the data points in Fig. $2 c$ were incorrect. The original and corrected versions are shown below.

The authors have also made some changes to the Supplementary Information: Fig. S9 has been replaced because the $x$ values were incorrect; the value of the carbonate buffer concentration has been corrected to $10 \mathrm{mM}$; and the sentence on page 2 that read "Subsequently, non-reacted aldehyde sites were blocked with bovine serum albumin (BSA, $0.1 \mathrm{mg} / \mathrm{mL})$ and ethanolamine (10 mM) in bicarbonate buffer for $1 \mathrm{~h}$." has been changed to "When modifying nanostars with antibodies, non-reacted aldehyde sites were blocked with bovine serum albumin (BSA, $0.1 \mathrm{mg} / \mathrm{mL})$ and ethanolamine $(10 \mathrm{mM})$ in bicarbonate buffer for $1 \mathrm{~h} . "$

These changes do not affect the results of the paper.

Original Fig. 2c

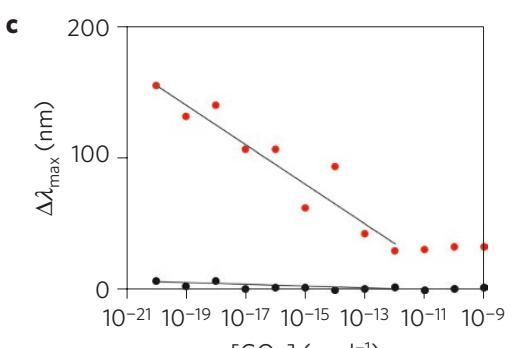

Corrected Fig. 2c

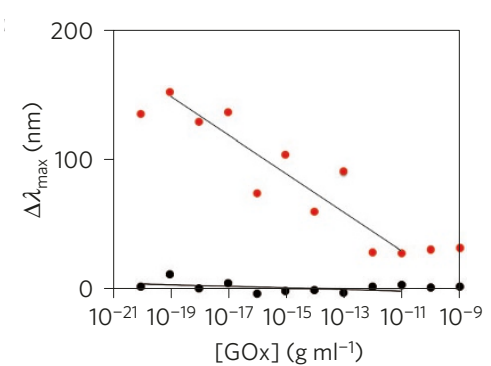

